\title{
Towards Geodesign: Repurposing Cartography and GIS?
}

\author{
Michael F. Goodchild, Ph.D. | good@geog.ucsb.edu
}

Center for Spatial Studies

Department of Geography

University of California, Santa Barabara

Santa Barbara, CA $93106-4060$

\section{REPLACE WORDCLOUD}

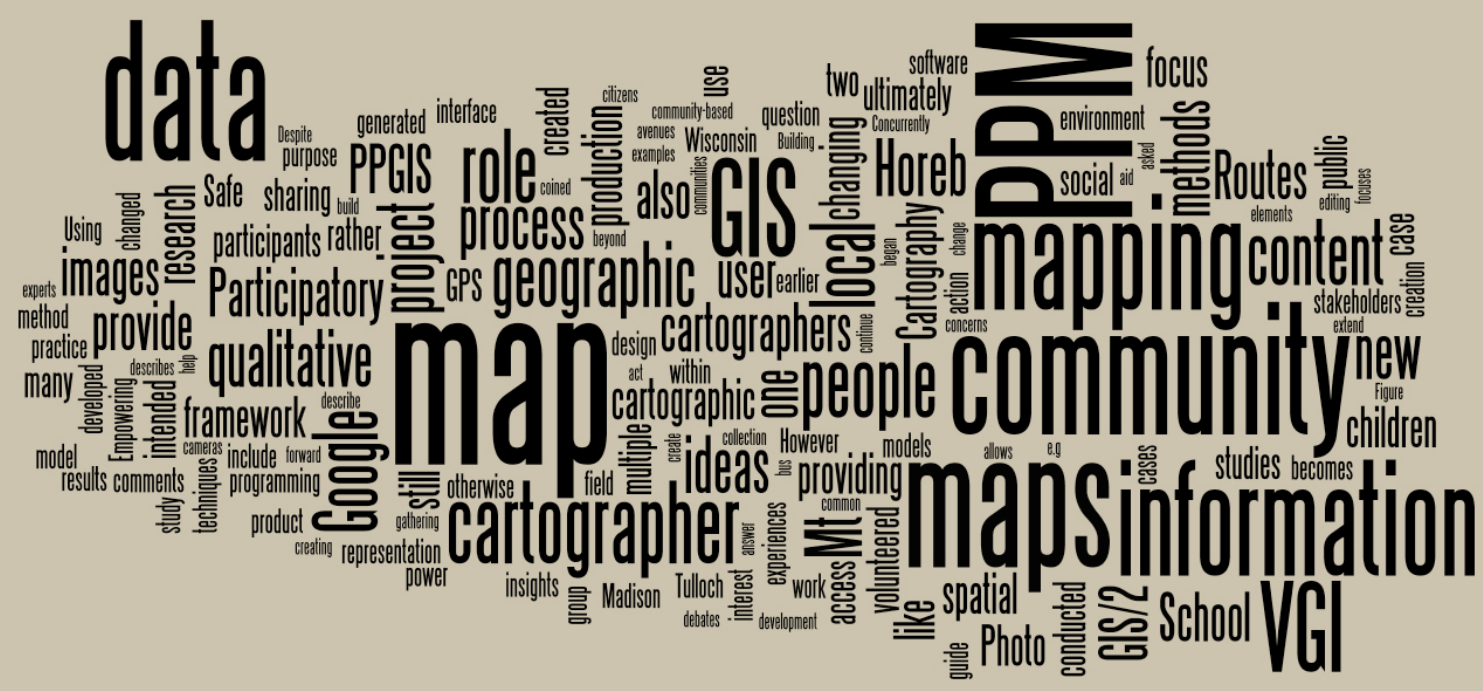

\section{ABSTRACT}

One of the original visions for GIS was as a tool for creating designs, but GIS has evolved in numerous other directions. Definitions of geodesign are reviewed, together with a short history of the concept. A distinction is drawn between Design and design, the latter being addressed through spatial decision support systems, and the former being seen as a superset of the latter. Geodesign also has a strong and well-defined relationship with cartography. The vision of landscape architecture propounded by the late Ian McHarg also provides a foundation for geodesign. Two existing gaps in the geodesign's computational tools are identified: support for sketch and implementation of models representing scientific knowledge of how the world works. Two important areas of research are identified that would address problems that currently impede geodesign.

\section{INTRODUCTION}

Although there are many historic roots of geographic information systems (GIS; Foresman 1998), one of the strongest lies in the notion of making design decisions by overlaying maps, each map representing one of the 
factors important in the decision. The net effect of each of the factors would be represented by the amount of light penetrating the layers at each point, allowing the decision maker to make an intuitive judgment as to the best solution. This is one of the central ideas of McHarg's (1969) Design with Nature, and the stack of layers has become an icon of the entire field of GIS, appearing on the front covers of many of its textbooks. One of the strongest arguments for GIS has been its ability to place such a simple and intuitive concept as overlaying transparent maps on a solid, reproducible-one might go so far as to say objective and scientific-footing. Abundant examples of this idea can be found in the field's textbooks, ranging from site selection for industrial plants to routing of power lines or highway corridors.

However, in the four decades that have elapsed since its birth, this notion of GIS as improving the process of design has become less central. GIS has evolved into a tool for performing spatial analysis in support of scientific discovery, a system for managing inventories of spatially distributed assets, a platform for automating the cartographic process and displaying information in map form, and a medium for communicating what is known about the surface and near-surface of the planet (Sui and Goodchild 2001). Yet we increasingly are aware of the planet's fragility, and of the need to make wise decisions about its future that are informed by evidence and by the best scientific knowledge. Now, more than ever, we need a technology of design that can work in tandem with human decision-making processes, bringing what we know about how the planet works to bear on the decisions that have to be made about its future. Humans have the power both to destroy the planet and to sustain it. We need tools that can predict for us the effects of tinkering with the Earth system, thus helping us to be effective stewards of the only planet we have.

This concept of science-based design sits at the interface among several disciplines. It involves the disciplines that traditionally have concerned themselves with design, including planning and landscape architecture. But it also involves the disciplines that acquire and accumulate fundamental knowledge about how environmental and social systems operate, including geography, ecology, hydrology, earth science, sociology, economics, and political science. Finally, it includes the new disciplines of information technology, especially geographic information science (GIScience; Goodchild 1992). Input from all three of these sets is needed if decisions are to be supported by welldesigned and powerful tools that are easy to use, and by the results of good science.

Over the past decade, there have been several discussions of the need to close what many have perceived as the growing gap between GIS and design. In January 2001, a workshop was held in Santa Barbara, California on Landscape Change, organized by a joint committee of landscape architects and GIScientists (http://www.ncgia.ucsb.edu/landscape/landscape.htm). A second workshop on Spatial Concepts in GIS and Design was held in Santa Barbara in late 2008 (http://www.ncgia.ucsb.edu/projects/scdg/). The term geodesign was suggested as a useful umbrella term for this examination of the common ground between GIS and design, with its implied emphasis on the 
geographic domain and geographic scales. Most recently, in January 2010 the first GeoDesign Summit was convened in Redlands, California (http:// www.geodesignsummit.com), bringing together GIS and design practitioners from academia, non-governmental organizations, government agencies, and the private sector, with over 150 participants from across a wide range of disciplines.

This paper presents one person's view of the nature of geodesign, of its objectives, of how the field might be conceptually framed, of its relationships to existing fields (especially cartography and GIS), and of research issues that need to be addressed if current impediments to effective geodesign are to be removed. The remainder of the paper is organized as follows. The second section reviews alternative definitions of geodesign, its domain of application, and its cognate disciplines. The third section discusses the McHarg vision and its limitations, updates it to the present day, and presents a brief critique. The fourth section describes the tools and computing environment that would be needed to support a fully-fledged practice of geodesign. The final section ends with some suggestions for future developments. The discussion is inevitably personal, with no implication that all possible topics and arguments have been covered. Nevertheless, the paper may provide a useful increment in our understanding of the nature of geodesign and of what needs to be done to move its agenda forward.

\section{WHAT IS GEODESIGN?}

\section{DEFINITIONS}

The GeoDesign Summit website quotes Carl Steinitz: "Geodesign is geography by design," a compellingly simple definition. If geography is the set of processes that operate on or near the Earth's surface, together with the forms that result from such processes, then geodesign is concerned with manipulating those forms and intervening in these processes to achieve specific objectives. Thus, it is normative in the sense that decisions are made about aspects of the geographic domain in order to achieve specified objectives, or norms. Normative efforts stand in contrast to the traditional aim of science, to discover general truths about the world; geodesign is interventionist in contrast to the more detached and dispassionate nature of pure science. Geodesign seeks to improve the world, whereas traditional science seeks only to provide the basis of knowledge on which the world might eventually be improved. Pure science is often carefully partitioned from application, and often sees its responsibilities as discharged when results have appeared in the pages of refereed journals. In that sense, geodesign lies within the domain of applied science and engineering, seeking ways of addressing practical problems using the scientific method.

Wikipedia defines geodesign as "a set of techniques and enabling technologies for planning built and natural environments in an integrated process, including project conceptualization, analysis, design specification, 
stakeholder participation and collaboration, design creation, simulation, and evaluation (among other stages)." The emphasis here is on built and natural environments, or Steinitz's geography, and also on the integration of the entire design process, presumably through technology.

Both of these definitions imply a very broad and traditional interpretation of the planning process. Others, however, have focused more on how planning can take advantage of the capabilities of GIS. Wikipedia also quotes Flaxman's address at the GeoDesign Summit: "Geodesign is a design and planning method which tightly couples the creation of design proposals with impact simulations informed by geographic contexts." In other words, the ability of modern GIS to create highly compute-intensive simulations of the effects of design scenarios provides an additional dimension to the traditional planning process, with its emphasis on visual display and intuition: geodesign is planning informed by scientific knowledge of how the world works, expressed in GIS-based simulations. In a similar, though less compute-intensive vein, and quoting Jack Dangermond from the GeoDesign Summit website, "Imagine if your initial design concept, scribbled on the back of a cocktail napkin, has the full power of GIS behind it. The sketch goes into the database, becoming a layer that can be compared to all the other layers in the database." Clearly, comparison of layers is only one of the multitude of functions that are easily invoked with today's GIS. Nevertheless, sketch and simulation provide two distinct notions of how the computational environment of a GIS might support geodesign.

One might also compare geodesign with other more widely recognized and traditional terms, such as computer-aided design (CAD). GIS has often been distinguished from CAD (Cowen 1988) by its emphasis on a geographic reference system, the richness of the attributes associated with features, its ability to deal with continuous fields (Couclelis 1992) in addition to discrete objects, and its rich set of analytic and modeling functions. In essence, the emphasis in $\mathrm{CAD}$ is on designing a structure through digital representation; in GIS it is on analyzing and modeling the structures present in the social and environmental worlds; and in geodesign it is on user-driven intervention in those worlds.

\section{SPATIAL OPTIMIZATION}

There is a long tradition of finding optimal solutions to design problems in the research domain known as spatial optimization. Much of this literature concerns finding optimal locations for point-like facilities, such as schools, fire stations, retail stores, or restaurants (Ghosh and Rushton 1987).

Numerous problems have been formulated, depending on the exact nature of the application, the objectives and constraints that apply, and the nature of the space within which optima are sought. For example, the field of locationallocation concerns the search for one or more locations for point-like facilities to serve a dispersed demand, and solutions involve both the optimal locations of the facilities and the service areas that each will cover. 
THE USER IS ABLE

TO INTERACT WITH

THE SOLUTION

VARIABLES IN

VARIOUS WAYS,

SUCH AS BY USING

SKETCH TOOLS TO

DEFINE OR EDIT

THEIR GEOMETRIES,

OR USING THE

KEYBOARD TO

DEFINE OR EDIT

THEIR ATTRIBUTES
More generally, spatial optimization problems can be characterized by the type of information represented by the solution. Some problems seek optimal locations for points, some for lines (e.g., transmission corridors), and some for areas (e.g., optimal allocation of land for specified uses). Some problems seek optimal allocations of one set of features to another, as in the case of optimal allocation of service areas for schools, or optimal patterns of transportation from origins to destinations. In general, a spatial optimization problem might find solutions in the form of any augmentation of a GIS database. From an object-oriented perspective (e.g., Zeiler 1999), this might mean the creation of a new feature class; the addition or deletion of features from an existing class; the addition or modification of one or more attributes of a feature class; the creation of a new association class representing patterns of interaction between existing origins and destinations; the creation of new routes that are themselves aggregations of an existing edge feature class, etc. In this way, the problems formulated as spatial optimizations can be related directly to the elements of a modern GIS database design. More broadly, we can see geodesign as transforming an existing database $\mathbf{D}$ into a new one D' through some combination of edits.

Spatial optimization provides a useful framework for geodesign, although it is often far too simplistic, as the next section explains. Spatial optimization requires an objective function that reflects the goals of the design, expressed in numeric form as a function of the solution variables that are available to be manipulated by the designer. It requires a solution space that is defined by the solution variables and limited by the constraints. The final design will occupy one point in the solution space. In geodesign, the solution variables all can be found in the database, as attributes or geometries of features, or as attributes of association classes. The user is able to interact with the solution variables in various ways, such as by using sketch tools to define or edit their geometries, or using the keyboard to define or edit their attributes.

\section{BIG-D AND SMALL-D DESIGN}

Spatial optimization often is seen as a task to be performed by a machine with no human intervention-as a fully automated edit of a database. Once the objectives and constraints are formulated, and the data are assembled, the machine is allowed to take over, producing a solution that by definition represents the best possible decision. It is often argued that such formal procedures provide a vast improvement over the messy, intuitive process of more traditional decision making. Disagreements between stakeholders over the objectives and constraints, or over the weights to be applied to different factors, can be handled through a variety of equally rigorous and mechanical multi-criteria problem formulations (Thill 1999). However, courts have sometimes held that a solution can be unacceptable against certain criteria, such as racial bias, even though the objective function and constraints included no such bias. Moreover, it may simply be naive to believe that human rationality, in the form of rigorously formulated optimization problems, can ever replace the messy nature of politics. Instead, spatial optimization is better seen as a collaboration between human and machine, 
in which the machine's role is simply to perform the calculations and iterations that humans find tedious - with the human still firmly in control.

This argument provides a useful basis for distinguishing between two visions of the design process. Small-d design takes a simplified view-design consists of the formulation of an optimization problem with objectives and constraints, the collection of data, the execution of a search for the optimum solution, and its implementation. In this somewhat naïve and simplistic view, implementation is seen as inevitable, because all participants agreed on the objectives and must therefore accept the result. Small-d design most commonly is associated with the disciplines of operations research, engineering, and management science.

Big-D Design sees the process as complicated by disagreements among stakeholders, difficulties in deciding what is optimal, feedback loops that modify objectives, constraints, and data as the process proceeds, and uncertainties about implementation. Figure 1, taken from the work of Steinitz (1990; Steinitz et al. 2003), structures Design as a sequence of six

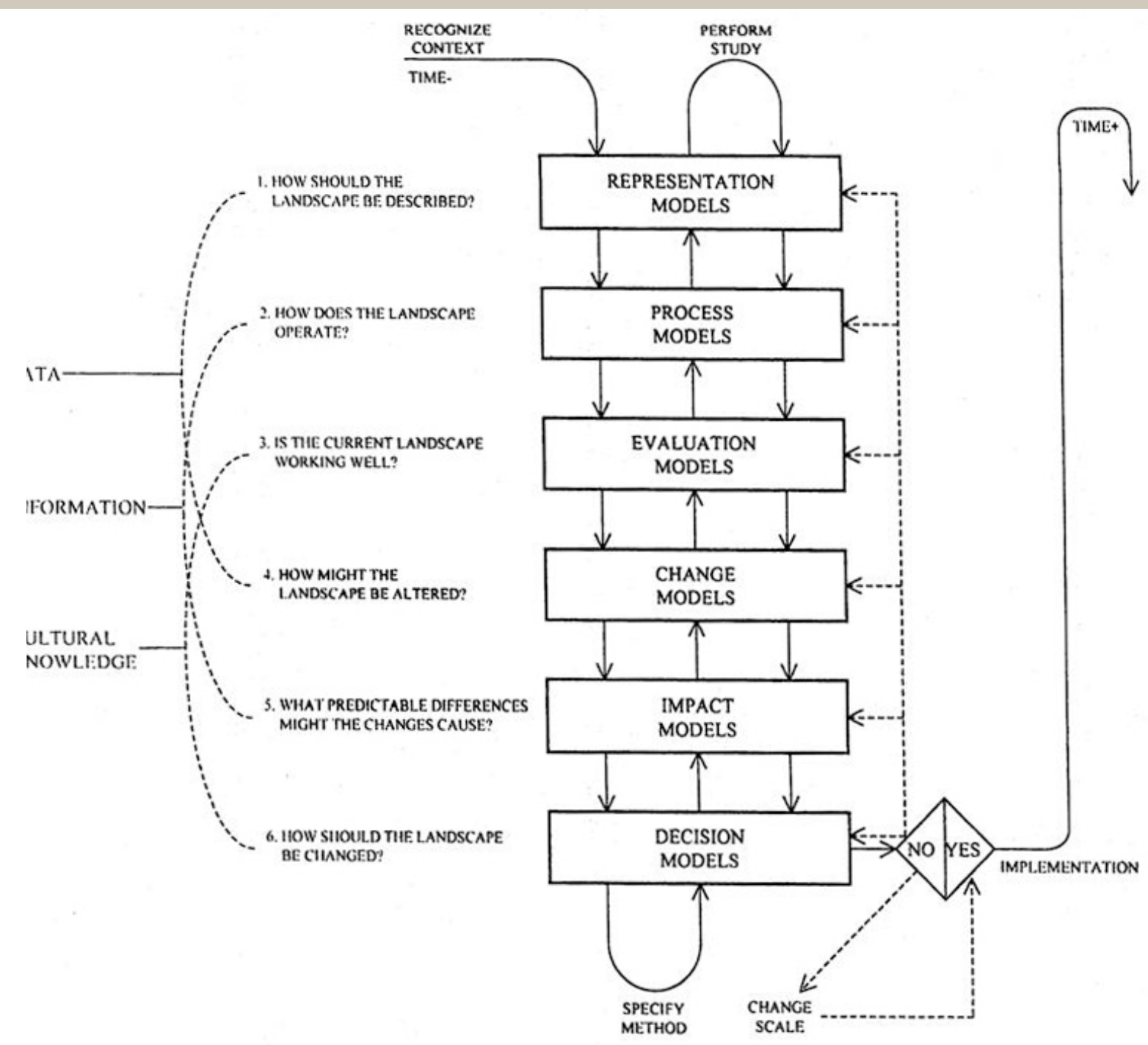


stages with iterative feedbacks - and similar schemata can be found in other sources. Each of these stages might be formalized as a model and supported by computational tools. Big-D Design most commonly is associated with the disciplines of landscape architecture and planning. As the dominant paradigm of geodesign, big-D Design — rather than small-d design—will be implied whenever the term design is used in the remainder of this paper.

The problem of conservation planning provides a useful illustration of the difference between design and Design. Conservation planning seeks to acquire a set of conservation areas in order to best preserve one or more biological species. A set of models is constructed from empirical data to predict the ability of a given parcel of land to support a given species. A spatial optimization problem is then formulated, seeking the best combination of land acquisitions to provide a sustainable population of the target species (Hof and Bevers 2002). Issues such as the connectedness of parcels are important to allow for interactions between breeding populations. The results can be expressed in the form of a new attribute of the landparcel feature class, denoting whether or not each land parcel is targeted for acquisition. Thorne, Cameron, and Quinn (2006) provide an example plan for land acquisitions in Southern California to preserve the mountain lion.

However, it is naïve to believe that the publication of such a plan will have no impact on the market for land, or on the attitudes of landowners. Instead, it commonly creates a strong and sustained reaction among the potentially affected landowners. Moreover, parcels inevitably will be acquired over an extended period of time, and it is likely that some of the optimal set will prove impossible to acquire, and will be replaced by alternative near-optimal parcels. In principle, each replacement affects the optimality of the entire solution, so the problem needs to be re-solved after every acquisition. In reality, then, the simple spatial optimization problem (design) is embedded in a much more complex process (Design) that is characterized by large amounts of uncertainty. Gallo (2007) shows how important it can be to avoid publishing a single, deterministic optimum solution, and instead suggests focusing on relative priorities expressed in probabilistic terms.

\section{GEODESIGN AND CARTOGRAPHY}

The display of geographic information in map form often is seen as an indispensable part of any geodesign process. Geodesign is by definition about geographic space, and Dangermond's definition quoted earlier points directly to cartography and the role of the computer as transforming an informal sketch into an element of a formal database. One of the achievements of GIS over the past 45 years has been the development of an integrated theory of geographic data representation, in other words, of a formal model of phenomena distributed over the surface and near-surface of the Earth. It includes discrete objects and continuous fields; points, lines, areas, and volumes; approaches to the representation of time; and solutions to the problem of representing flows and interactions (Goodchild, Yuan, and Cova 2007). GIS has progressed substantially beyond the earlier map 
metaphor, when a GIS was viewed informally as a computer containing maps (Goodchild 1988). Animation, for example, is now almost routine in computerized displays, but was impossible as long as maps were confined to analog form on paper.

A two-dimensional display of geographic data, with the defining dimensions of the display representing (mapping in its mathematical sense) the spatial dimensions of the data, is a powerful way of showing the user what is present at every location within the extent of the display. Every point on a paper map can be printed with any color, and similarly every pixel on a computer display can be programmed to display any color. The visual impression of linear features is created by using a similar color along a linear sequence of points or pixels, and similarly an area is visualized by displaying its boundary, or by filling it with a uniform color or pattern. Annotation is communicated by linking points or pixels into the form of characters.

Nevertheless, it has always been difficult to use this approach to display geographic information that concerns points taken two at a time-in other words, relationships or interactions between mapped features (Takeyama and Couclelis 1997). It is difficult, for example, to label a feature on a map according to the several names given to it by the inhabitants of neighboring areas, or to show flows of migrants or telephone calls. Such flows occur between pairs of features (origins and destinations), are not independent properties of either, and therefore cannot be displayed by symbolizing either feature alone, or even in combination. Lines might be drawn to connect origins and destinations, and appropriately symbolized, but in many cases the actual path of flow is not known (Glennon 2010), and a large number of such lines can render the map unreadable. In the previous section, this type of information was characterized as an association class in the objectoriented paradigm. In short, while the results of any spatial optimization can be regarded as a modification of a database, not all such modifications are equally easily visualized cartographically. Sketch is an important way of communicating simple geographic features between user and GIS, and other important kinds of input can also be captured through interaction between the user and a map display. More broadly, cartography is a useful part of geodesign, but not all aspects of geodesign are inherently cartographic.

\section{THE MCHARG VISION}

Reference has already been made to the early days of GIS and the importance of design based on multiple layers of input. McHarg's vision for his school of landscape architecture at the University of Pennsylvania used the stack of layers as a metaphor for the organization of the school (McHarg 1996). Each layer corresponded to one discipline whose subject matter was important to landscape design, including ecology, hydrology, and geology. Overlaying the layers symbolized the simultaneous attention that needed to be paid to each of these as a plan was developed. Each layer would be shaded according to the weight to be assigned to the corresponding discipline's 
issues, and the relevant factors present at each point.

This is only one aspect of McHarg's contribution, of course, but it is emphasized here because of the way it links design and GIS, and thus relates to the topic of this paper. Suppose, for example, that the impact of a proposed pipeline at location $\mathbf{x}$ is determined to be $\mathrm{z}_{\mathrm{e}}(\mathrm{x})$ per unit area, when measured from the perspective of ecology. Similarly, the impact of the pipeline at $\mathbf{x}$ from the perspective of hydrology might be $z_{h}(x)$ per unit area, and the economic cost of acquiring the necessary land might be $\mathrm{z}_{\mathrm{c}}(\mathrm{x})$. The three measures are incommensurate, of course, so weights must be assigned to reduce them to a common metric, and to rate their relative importance. Define these weights as $\mathrm{w}_{\mathrm{e}}, \mathrm{w}_{\mathrm{h}}$, and $\mathrm{w}_{\mathrm{c}}$ respectively. Then the problem can be formulated as finding a route such that the total weighted cost along the route is minimized. If $S$ denotes the solution set, that is, the set of points along the route, then the task is to minimize:

$$
Z=\sum_{\mathbf{x} \in S}\left[w_{e} z_{e}(\mathbf{x})+w_{h} z_{h}(\mathbf{x})+w_{c} z_{c}(\mathbf{x})\right]
$$

In the analog method described in Design with Nature (McHarg 1969), both $w$ and $z$ must be captured by the darkness of the corresponding layer at point $\mathbf{x}$, and the optical process of overlaying layers replaces the summation in the equation by a multiplication. In reality, of course, the kind of rigor exhibited in the equation was never intended to be imposed in the analog method, but the more formal GIS overlay process forces the user to address all of these issues explicitly. For example, the weights $w$ might be assigned using Saaty's Analytical Hierarchy Process (AHP), or a variety of other methods that are described in the standard texts on multicriteria decision analysis (Malczewski and Rinner 2010; Thill 1999). Similarly, it is common to address the "applesand-oranges" issue of non-commensurate variables by normalizing each to the range 0 to 1 . But since the observed range depends on the exact extent of the study area, there are obvious logical flaws in this practice.

Despite the informality of the analog model, McHarg clearly intended his design process to be informed by science, and achieved this by constructing each layer according to the knowledge base of the corresponding discipline. Moreover, the school included representatives of each of those disciplines on its staff, forcing intensive engagement and interaction. This is a very different approach from that commonly followed in most universities, where the science disciplines are separated from the design disciplines, often across the boundaries between colleges or faculties. One of the underlying themes of geodesign is its potential to reduce that separation.

McHarg's vision is now more than four decades old, so it makes sense to ask whether and how it should be updated to the present. Enormous advances have been made in the disciplines that study social and environmental processes on the Earth's surface, and an argument can clearly be made for including all of them to the extent that they are relevant to a specific design question. But in addition, we know far more now than we did then about the 
process of decision making, and particularly about the role of uncertainty. Thus, it would seem important to include decision scientists and statisticians in the mix, especially spatial statisticians. We also would need to include the computer scientists and information scientists who address issues of representation and develop the algorithms needed to implement scientific knowledge - especially geographic information scientists—and the experts in remote sensing and sensor networks who address issues of spatial data acquisition. We also would need to include the cartographers and specialists in spatial cognition who address human factors in the interactions between designers and tools, and the social psychologists who study processes of group interaction.

\section{COMPUTATIONAL SUPPORT FOR GEODESIGN}

In the complex process represented by Figure 1, geodesign can be partitioned into a series of stages, each underlain by a model and each supported by computational tools. This is very different from the conceptualization of small-d design, in which the entire process occurs in a single stage, and in which a large proportion of control is surrendered to the computational system and its task of finding the best solution. The field of spatial decision support systems (SDSS) has long addressed the kinds of computational tools needed to support design decisions, and has accumulated a substantial literature (Leung 1997; Sugumaran and Degroote 2010). Li and her collaborators have recently constructed a very substantial collection of Web resources (http://www.institute.redlands.edu/sds), including an ontology of SDSS, in an effort to address the varying use of terms and to clarify the field's relationship with other cognate fields.

What then is the relationship between SDSS and geodesign? SDSS has its roots in the early 1990s (Densham and Goodchild 1990), and in a desire to apply GIS tools to a host of problems of spatial optimization. SDSS has always had a strong science base, so one might see geodesign as an effort to expand SDSS to include some of the design problems that have traditionally made less use of scientific knowledge to simulate the effects and impacts of plans. In other words, traditional SDSS may best be seen as a subset of geodesign, if any distinction is needed.

Reference was made earlier to the notion that there are two areas where geodesign tools need further development:

\section{SKETCH TOOLS}

The first key area of support for geodesign is sketch, or the ability of the user to create informal renderings of points, lines, and areas in geographic space, and to have the computational system capture, formalize, and store these. ESRI's ArcSketch already offers some of these capabilities, and Google's 
SketchUp extends them to the third spatial dimension.

In essence, sketch tools would allow the user to edit a GIS database by inserting new point, line, and area features. These might be added to existing feature classes or might be captured as entirely new classes. For example, a user seeking the best locations for a number of new retail stores to add to an existing chain might sketch potential locations. The system then might evaluate these locations based on a predictive model of store sales, or use them as the starting points for an optimal search procedure. From this perspective, sketch tools are augmentations of existing GIS database editing functions. But the emphasis is rather different; in conventional editing, the user seeks to approximate a truth, as expressed perhaps by a source document, whereas no such truth exists in the case of sketch, which is inherently vague.

IN CONVENTIONAL EDITING, THE USER SEEKS TO APPROXIMATE A TRUTH, AS EXPRESSED PERHAPS BY A SOURCE DOCUMENT, WHEREAS NO SUCH TRUTH EXISTS IN THE CASE OF SKETCH, WHICH IS INHERENTLY VAGUE

\section{SIMULATION TOOLS}

The second area is simulation, or the examination of design scenarios by simulating their impacts based on sound scientific knowledge. For example, the impacts of a proposed new highway might be examined by simulating its effects on the pattern of traffic in the surrounding area; on the downstream effects on local hydrology; and on noise and atmospheric pollution. Calibrated models exist for each of these sets of impacts as a result of basic scientific research. Moreover, in many areas large numbers of models exist, based on different sets of assumptions, requiring different inputs, and yielding different answers. One of the most valuable outputs of a GIS simulation may lie in the uncertainties associated with predictions, based on uncertainties within each model and on variation across models.

Many successful efforts have been made to integrate models of social and environmental processes into GIS, and the results are described in a substantial literature (Goodchild, Parks, and Steyaert 1993; Skidmore 2002). Many models are difficult to integrate with GIS and with other models because of lack of standards governing data formats, and there is also a need for greater standardization in the languages in which model software is written. Both of these factors impede the goals of geodesign, because they make it difficult to implement many models as simple functions of a geodesign software environment.

More specifically, research is needed to address two issues of major importance:

1. Models need to be encapsulated easily within GIS, so that they can be executed and the results analyzed within the workflow of a geodesign process. This implies that data inputs and outputs need to follow GIS data format standards so they can be integrated readily with GIS databases, and that model parameters be exposed to the user through a GIS interface.

2. Models need to be written in a common language, so that their component parts can be reassembled and reused readily. In practice, 
models are commonly written in a range of computing environments, from source languages such as $\mathrm{C}++$ to scripting languages such as Python. Efforts to develop a common, uniform language for GIS have made only limited progress in the past (e.g., the Map Algebra of Tomlin 1990), though van Deursen's scripting language for PCRaster (van Deursen 1995) offers a comprehensive solution at least for simulations over a cellular landscape. A comprehensive solution to this problem would be a major contribution to the goals of geodesign.

Behind this need for a common language lies a much more fundamental problem, that of defining a standard set of GIS operations. While various taxonomies have been published, it is regrettably true that after 45 years of GIS development there exists no standard set that is defined on a rigorous conceptual basis. Instead, the sets of functions offered by popular GIS packages, such as the ArcToolbox, are the result of a haphazard historical process of development. There are no universal standards and no rigorous concept of granularity, making it difficult to discover functions offered on the Web and undermining the entire concept of service-oriented architecture. A conceptual framework for the structuring of GIS functionality would be an enormously valuable contribution, enabling a new level of interoperability across the GIS field.

\section{DISCUSSION AND CONCLUSION}

It will be obvious from the preceding sections that geodesign is not new, but instead represents a re-examination and perhaps a repurposing of a number of established fields. In the case of GIS, this re-examination is prompted by a perceived lack of attention to the use of GIS in design, and to its potential role in improving the geographic world. In the case of spatial optimization, it is prompted by the perception that design problems are more complex than simple mathematical formulations, and that the political process of decision making is more complex than the execution of a single optimization. In the case of landscape architecture, it is prompted by the notion that science can play a much stronger role in informing important decisions over the use of land, and that GIS is a valuable platform for integrating scientific knowledge into the design process.

The design of tools is driven by a constant tension between the specific and the general: between the scale economies that result from a one-size-fits-all solution, and the speed with which a targeted solution to a specific problem can be constructed. In the 1970s, GIS emerged as a generic solution to a set of requirements that ranged from cartographic editing to land-use planning and the administration of the census. Today, a suite of integrated geodesign tools may emerge from the realization that a host of geographic design problems share a common structure, and rely on access to a common GIS database. Just as with GIS, the attendant economies of scale in software

TODAY, A SUITE

OF INTEGRATED GEODESIGN TOOLS MAY EMERGE FROM THE REALIZATION THAT A HOST OF GEOGRAPHIC DESIGN PROBLEMS SHARE A COMMON STRUCTURE, AND RELY ON ACCESS TO A COMMON GIS DATABASE 
production, training, and documentation would be enormous.

For that to happen, however, several issues have to be resolved. Two have already been mentioned: the lack of interoperability between existing model codes, and the lack of a language within which an integrated vision of reusable codes could be implemented. In addition, however, it is important to address the question of how a suitable computing environment, once defined, might be widely adopted. Several successful models can be found in the history of GIS:

NOW MORE THAN EVER, WE SENSE THE NEED FOR EFFECTIVE TOOLS THAT CAN HELP US TO ENSURE A DESIRABLE FUTURE FOR THE PLANET, AND GIS CLEARLY CONTAINS THE FOUNDATION FOR SUCH TOOLS
1. The commercial software route. Commercial software developers have the development staffs and the necessary mechanisms for promotion, training, and support to turn a design into a widely adopted reality. An advisory group of geodesigners might define the framework, and ensure that it was successfully implemented in tools.

2. The open-source route. GRASS was an early and highly successful effort to develop a comprehensive GIS for environmental modeling, based on open-source code and a network of researchers who added routines within a loosely defined set of standards. The role of the US Army Corps of Engineers in providing the initial foundation was critical, and suggests that a suitable strategy would be to obtain a major grant from a funding agency to construct the framework and to build the initial community of contributors and users.

\section{The research center route. $\mathrm{GeoDa}$ (http://geodacenter.asu.edu) is another} example of a highly successful package of tools, in this case addressing the needs of social scientists for easy-to-use software for spatial analysis. It was developed under a major center grant from the US National Science Foundation, which funded not only the code but also tutorials and workshops that publicized its applications.

Design was clearly an early objective of GIS, but as argued earlier, it tended to lose its centrality as GIS evolved to serve more lucrative and immediate markets. Now more than ever, we sense the need for effective tools that can help us to ensure a desirable future for the planet, and GIS clearly contains the foundation for such tools. The concept of geodesign presents a simple banner for a renewed effort to emphasize the value of cartography and GIS as tools for improving and sustaining the surface and near-surface of the Earth.

\section{ACKNOWLEDGEMENT}

This paper has benefited from numerous discussions over the past few months, most notably with Bill Miller (ESRI), Naicong Li (Redlands Institute), and Carl Steinitz (Harvard University). The opinions and conclusions expressed are entirely those of the author, however. 


\section{REFERENCES}

Couclelis, H. 1992. People manipulate objects (but cultivate fields): beyond the raster-vector debate in GIS. In A.U. Frank and I. Campari, eds., Theories and Methods of Spatio-Temporal Reasoning in Geographic Space. Lecture Notes in Computer Science 639:65-77. Berlin: Springer.

Cowen, D.J. 1988. GIS versus CAD versus DBMS: What are the differences? Photogrammetric Engineering and Remote Sensing 54:1551-1554.

Densham, P.J. and M.F. Goodchild. 1990. Research Initiative 6: Spatial decision support systems: report for the specialist meeting. Technical Report, 90-5. Santa Barbara, CA: National Center for Geographic Information and Analysis.

Foresman, T.W. 1998. The History of Geographic Information Systems: Perspectives from the Pioneers. Upper Saddle River, NJ: Prentice Hall PTR.

Gallo, J. 2007. Engaged conservation planning and uncertainty mapping as a means towards effective implementation and monitoring. $\mathrm{PhD}$ diss., University of California, Santa Barbara.

Ghosh, A. and G. Rushton, eds. 1987. Spatial Analysis and LocationAllocation Models. New York: Van Nostrand Reinhold.

Glennon, J.A. 2010. Creating and validating object-oriented data models: modeling flow within GIS. Transactions in GIS 14(1): 23-42.

Goodchild, M.F. 1988. Stepping over the line: technological constraints and the new cartography. American Cartographer 15:311-319.

Goodchild, M.F. 1992. Geographic information science. International Journal of Geographical Information Systems 6(1): 31-45.

Goodchild, M.F., B.O. Parks, and L.T. Steyaert, eds. 1993. Environmental Modeling with GIS. New York: Oxford.

Goodchild, M.F., M. Yuan, and T.J. Cova. 2007. Towards a general theory of geographic representation in GIS. International Journal of Geographical Information Science 21(3): 239-260.

Hof, J.G. and M. Bevers. 2002. Spatial Optimization in Ecological Applications. New York: Columbia University Press.

Leung, Y., 1998. Intelligent Spatial Decision Support Systems. Berlin: Springer Verlag.

Malczewski, J. and C. Rinner. 2010. Multicriteria Decision Analysis in Geographic Information Science. Berlin: Springer.

McHarg, I.L. 1969. Design with Nature. Garden City, NY: Natural History Press. 
McHarg, I.L. 1996. A Quest for Life: An Autobiography. New York: Wiley.

Skidmore, A. 2002. Environmental Modelling with GIS and Remote Sensing. New York: Taylor and Francis.

Steinitz, C. 1990. A framework for theory applicable to the education of landscape architects (and other environmental design professionals). Landscape Journal 9:136-143.

Steinitz, C., H. Arias, S. Bassett, M. Flaxman, T. Goode, T. Maddock, D. Mouat, R. Peiser, and A. Shearer. 2003. Alternative Futures for Changing Landscapes: The San Pedro River Basin in Arizona and Sonora. Washington, DC: Island Press.

Sugumaran, R. and J. Degroote. 2010. Spatial Decision Support Systems. Boca Raton, FL: CRC.

Sui, D.Z. and M.F. Goodchild. 2001. Guest Editorial: GIS as media? International Journal of Geographical Information Science 15(5): 387-389.

Takeyama, M. and H. Couclelis. 1997. Map dynamics: integrating cellular automata and GIS through Geo-Algebra. International Journal of Geographical Information Science 11:73-91.

Thill, J.-C. 1999. Spatial Multicriteria Decision Making and Analysis: A Geographic Information Sciences Approach. Brookfield, VT: Ashgate.

Thorne, J.H., D. Cameron, and J.F. Quinn. 2006. A conservation design for the central coast of California and the evaluation of mountain lion as an umbrella species. Natural Areas Journal 26:137-148.

Tomlin, C.D. 1990. Geographic Information Systems and Cartographic Modeling. Englewood Cliffs, NJ: Prentice Hall.

van Deursen, W.P.A. 1995. Geographic Information Systems and Dynamic Models: Development and Application of a Prototype Spatial Modelling Language. PhD Dissertation, Utrecht University.

Zeiler, M. 1999. Modeling Our World: The ESRI Guide to Geodatabase Design. Redlands, CA: ESRI Press. 